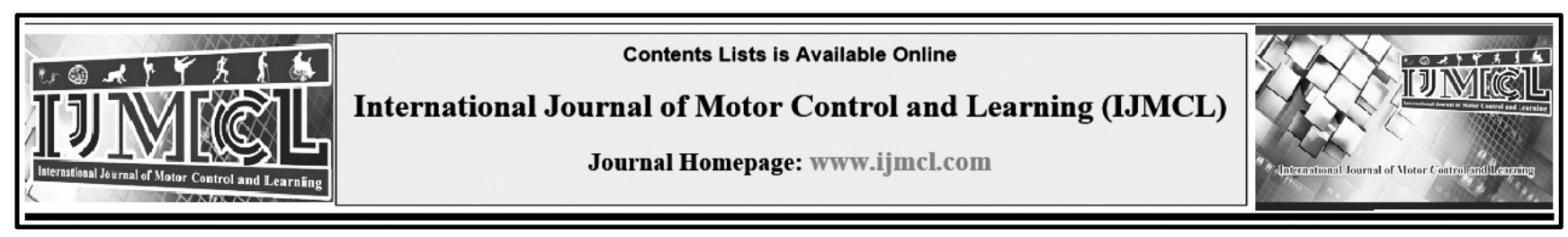

\title{
The Need to Psychological Screening of Para Athletes
}

\author{
Mohammad Vaez Mousavi ${ }^{a *}$ \\ ${ }^{\text {a }}$ Professor of Sport and Exercise Psychology, National Olympic and Paralympic Academy, Tehran, Iran. \\ *Corresponding E-mail: mohammadvaezmousavi@chmail.ir \\ Received: 2020/04/25 \\ Accepted: 2020/07/09 \\ Published: 2020/08/03
}

\section{DEAR EDITOR}

As the level of practice or competition increases, the risk of negative incidents similar to injury, overtraining, burnout, anxiety, and eating disorders also increases, which affects para athletes' health and wellbeing (Kenta and Corban, 2014). Most athletes need to control their chronic pain, prevent sport injuries, and cope with sleep disturbances (Martin, 2017). Para athletes face challenges in air travelling, living in the camps or game villages, and city transportations (Campbell and Jones, 2002) which may cause psychological stress. Sometimes, gender, would be also a challenge. Comparing male to female para athletes, it was found that females face more cultural challenges; in some cultures, female sport is not of significance and is not considered as part of feminine values and competences (Shakespeare, Gillespie-Sells, Davies, 1996).

Most of psychological studies on para sports examines the advantages of sport activities for people with disability; however, to improve para athletes' wellbeing and mental health, it will be necessary to look out for both psychological excellences which para athletes may develop by participating in para sports, and also psychological characteristics which para athletes need to develop to be able to cope with para sports' challenging situations.

Sport psychologists are employed during or right before competition season to improve para athletes' performance; they teach para athletes to use mental skills and to cope with stress inducing situations. Our last 12 years of experience in Iran NPC indicates that this is not sufficient. A significant percent of athletes is still suffering from anxiety, depression, sleep disturbance, and communication problems. Several other factors like changes in classification rules, changes in competitions timetables, lack of knowledge about medicines/supplements, change of coaching staff and so on, cause stress for para athletes all year round. Therefore, constant monitoring of para athletes' psychological states seems necessary.

Creating a psychological profile for every para athlete who attends a national team camp will be beneficial in several senses:

○ In depth interview with the para athlete provides important psychological states and traits;

- Data collected in the profile will help the newly employed sport psychologists to put emphasis on the most important issues;

- And finally, these data will help to signify brief contact consultations during competitions.

Given the significance of psychological profiling of para athletes, it is duly expected that the IJMCL put special emphasis on publishing papers related to their psychological status. This may in turn encourages investigators in the vast field of sport and exercise psychology to put extra effort in investigating psychological aspects of para athletes, related to their mental health and also to their performance. 


\section{References}

1. Kenttä, G., \& Corban, R. (2014). Psychology within the Paralympic Context-Same, Same or Any Different? Olympic Coach, 25(3), 15-25.

2. Martin, J. J. (2017). Oxford Research Encyclopedia of Psychology, Edition: 1st, Chapter: Psychological Considerations for Paralympic Athletes, Publisher: Oxford University, Editors: Edmund Acevedo, pp.1-27.

3. Campbell, E., \& Jones, G. (2002). Sources of stress experienced by elite male wheelchair basketball players. Adapted Physical Activity Quarterly, 19(1), 82-99.

4. Shakespeare, T., Gillespie-Sells, K. and Davies, D. (1996). The sexual politics of disability: Untold desires London: Cassell. 